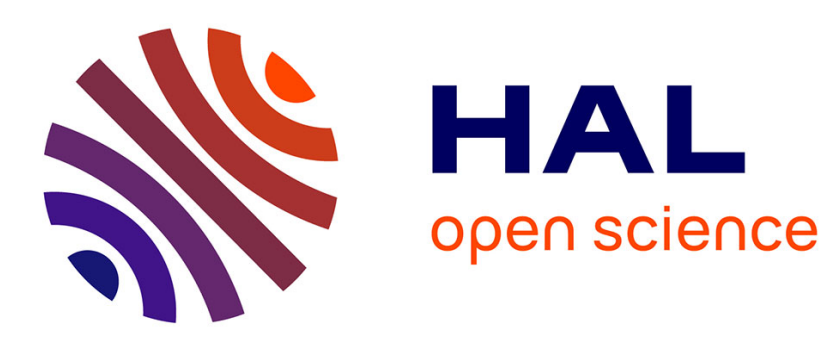

\title{
Pertes de poids du canard de Barbarie mâle lors du jeûne avant abattage
}

B. Leclercq, H. de Carville, G. Guy

\section{To cite this version:}

B. Leclercq, H. de Carville, G. Guy. Pertes de poids du canard de Barbarie mâle lors du jeûne avant abattage. Productions Animales, 1989, 2 (5), pp.353-356. hal-00895883

\section{HAL Id: hal-00895883 https://hal.science/hal-00895883}

Submitted on 1 Jan 1989

HAL is a multi-disciplinary open access archive for the deposit and dissemination of scientific research documents, whether they are published or not. The documents may come from teaching and research institutions in France or abroad, or from public or private research centers.
L'archive ouverte pluridisciplinaire HAL, est destinée au dépôt et à la diffusion de documents scientifiques de niveau recherche, publiés ou non, émanant des établissements d'enseignement et de recherche français ou étrangers, des laboratoires publics ou privés. 
INRA Prod. Anim.. 1989, 2 (5), 353-356
B. LECLERCQ, H. de CARVILLE, G. GUY

INRA Station de Recherches Avicoles 37380 Nouzilly

\section{Pertes de poids du canard de Barbarie mâle lors du jeûne avant abattage}

Les pertes de poids des animaux entre leur mise à jeun et l'abattage constituent un élément des transactions commerciales entre l'éleveur et l'abattoir. La connaissance de ces pertes et des facteurs susceptibles de les modifier présente un intérêt indéniable. Or on dispose de très peu d'information sur ce sujet. Pour le poulet de chair un travail réalisé en Hollande (Verkamp 1985) a abouti à une équation permettant d'estimer la baisse du poids vif en fonction du sexe, de la présence ou de l'absence d'abreuvement et du temps. Chez le canard de Barbarie, production avicole en pleine expansion, aucune information n'a été publiée sur ce sujet. Les deux expériences rapportées ci-après ont pour objet de combler cette lacune. Les enseignements qu'on peut en tirer pourront servir de base à des accords contractuels entre éleveurs et abattoir, et permettront également de corriger les performances enregistrées en diverses circonstances pour rendre leur comparaison plus exacte.

Au cours des deux expériences, on a distingué le poids vif à la mise à jeun et le poids vif à la mise en cages. Il s'écoule le plus souvent 8 heures entre ces deux opérations; durée qui correspond au transit intestinal et à l'excrétion de la majeure partie de la fraction indigestible de l'aliment. Ensuite les animaux en cages sont transportés vers l'abattoir. La durée du transport et l'attente avant l'abattage sont alors l'occasion de pertes de poids supplémentaires.

\section{Résumé}

Au cours de deux essais on a mesuré la perte de poids vif de canards de Barbarie en fonction de plusieurs paramètres : présence ou absence d'abreuvement, pesée en cages de transport ou individuelle, température ambiante. Les animaux ont été mis à jeun pendant 8 heures puis placés en cages de transport. La reproductibilité des pertes au cours des 8 premières heures de jeûne est médiocre. En revanche les pertes de poids en cages sont très reproductibles. Elles peuvent être considérées comme linéaires en fonction du temps; la décroissance étant de $0,20 \%$ par heure. Une température ambiante de l'ordre de $20^{\circ} \mathrm{C}$ accentue ces pertes par rapport à celle de $10^{\circ} \mathrm{C}$. L'abreuvement n'exerce aucune influence significative.

\section{Expérience 1}

Quarante-huit canards de Barbarie (Grimaud R51), de sexe mâle, âgés de 83 jours, ont été pesées individuellement à $8 \mathrm{~h}$, puis laissés sur litière, à jeun et sans eau. Ils sont demeurés ainsi pendant 8 heures puis ont été à nouveau pesés et placés en cages de transport plastique (dimensions $85 \mathrm{~cm} \times 65 \mathrm{~cm} \times 29 \mathrm{~cm}$ ) à raison de 4 canards par cage. Toutes les cages (12) ont été pesées, 2,4 et 12 heures après la mise en cages des animaux, soit 10, 12 et 24 heures après la mise à jeun. En outre les animaux de 5 cages ont été pesés individuellement à chaque stade, de façon à comparer la perte de poids en cages à la perte de poids réelle des animaux. L'ensemble de cet essai s'est déroulé à l'intérieur du bâtiment d'élevage à une température voisine de $18^{\circ} \mathrm{C}$.

\section{Expérience 2}

Quarante canards de sexe mâle (Grimaud R41), âgés de 91 jours ont été répartis en 2 lots de 20. Ils ont tous été pesés individuellement à $7 \mathrm{~h}$ le matin puis mis à jeun avec eau ou sans eau en cage sur fond grillagé de $1,3 \mathrm{~m} \times 1 \mathrm{~m}$. 
Huit heures plus tard les canards ont de nouveau été pesés puis mis en cages de transport. Celles-ci contenant les canards sont pesées. Elles sont réparties par moitié dans un local où la température est de $11^{\circ} \mathrm{C}$ et pour l'autre moitié à $23^{\circ} \mathrm{C}$; ces températures n'ont pas été régulées mais enregistrées tout au long de l'expérience. Les cages pleines et les oiseaux individuellement sont pesés 3,6 et 16 heures après le début de la mise en cage, soit 11,14 et 24 heures après le début du jeûne.

\section{Résultats}

Le poids vif moyen des canards décroît avec le temps (figure 1). Exprimés en valeurs relatives par rapport au poids vif à la mise à jeun ou par rapport à la mise en cage (tableau 1) les poids vifs présentent des valeurs dont on peut analyser la forme de la courbe de décroissance. Quand les animaux sont pesés individuelle- ment, cette décroissance est significativement différente d'une droite dont la pente est légèrement inférieure à celle des animaux pesés individuellement (figure 1).

Les résultats de la seconde expérience sont rapportés dans le tableau 2. La forme des courbes de décroissance des poids vifs (figure 2) exprimés en valeur relative par rapport au poids initial (à la mise à jeun ou à la mise en cage) ne s'écarte pas significativement de la linéarité, sauf à $23^{\circ} \mathrm{C}$ pour des canards mis à jeun avec eau. Dans nos conditions le fait de laisser de l'eau à la disposition des animaux pendant les 8 premières heures de jeûne n'influence pas la forme ni la vitesse de la décroissance des poids vifs. En revanche la température du local dans lequel sont placées les cages exerce un effet qui est à la limite de la signification statistique; on remarque qu'à $23^{\circ} \mathrm{C}$ les pertes de poids sont un peu plus prononcées qu'à $11^{\prime \prime} \mathrm{C}$.

Tableau 1. Evolution du poids vif des canards de Barbarie au cours du jeûne (Expérience 1).

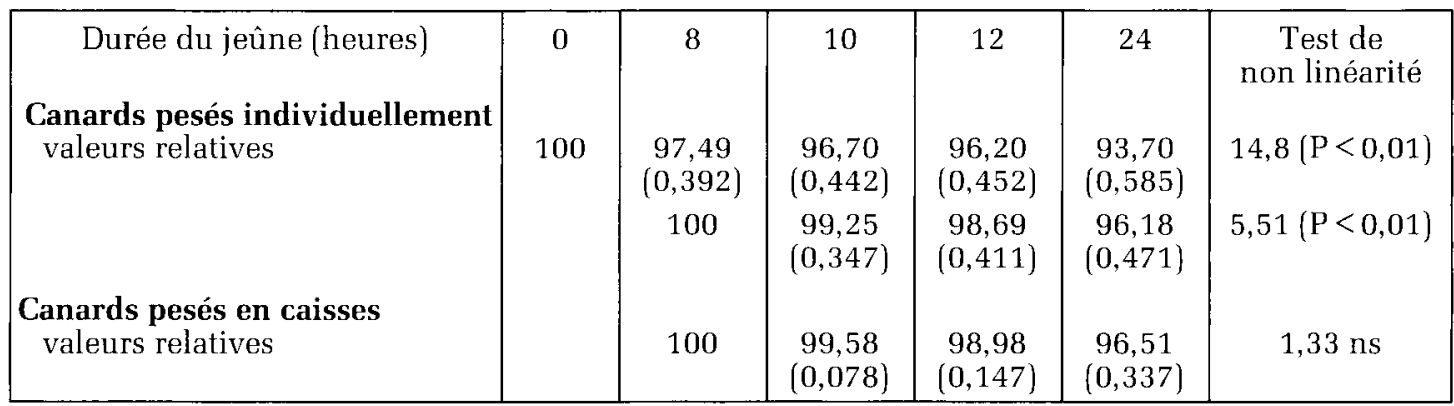

Entre parenthèses sont présentés les écarts-types.

Tableau 2. Evolution du poids vif des canards de Barbarie au cours du jeûne (Expérience 2).

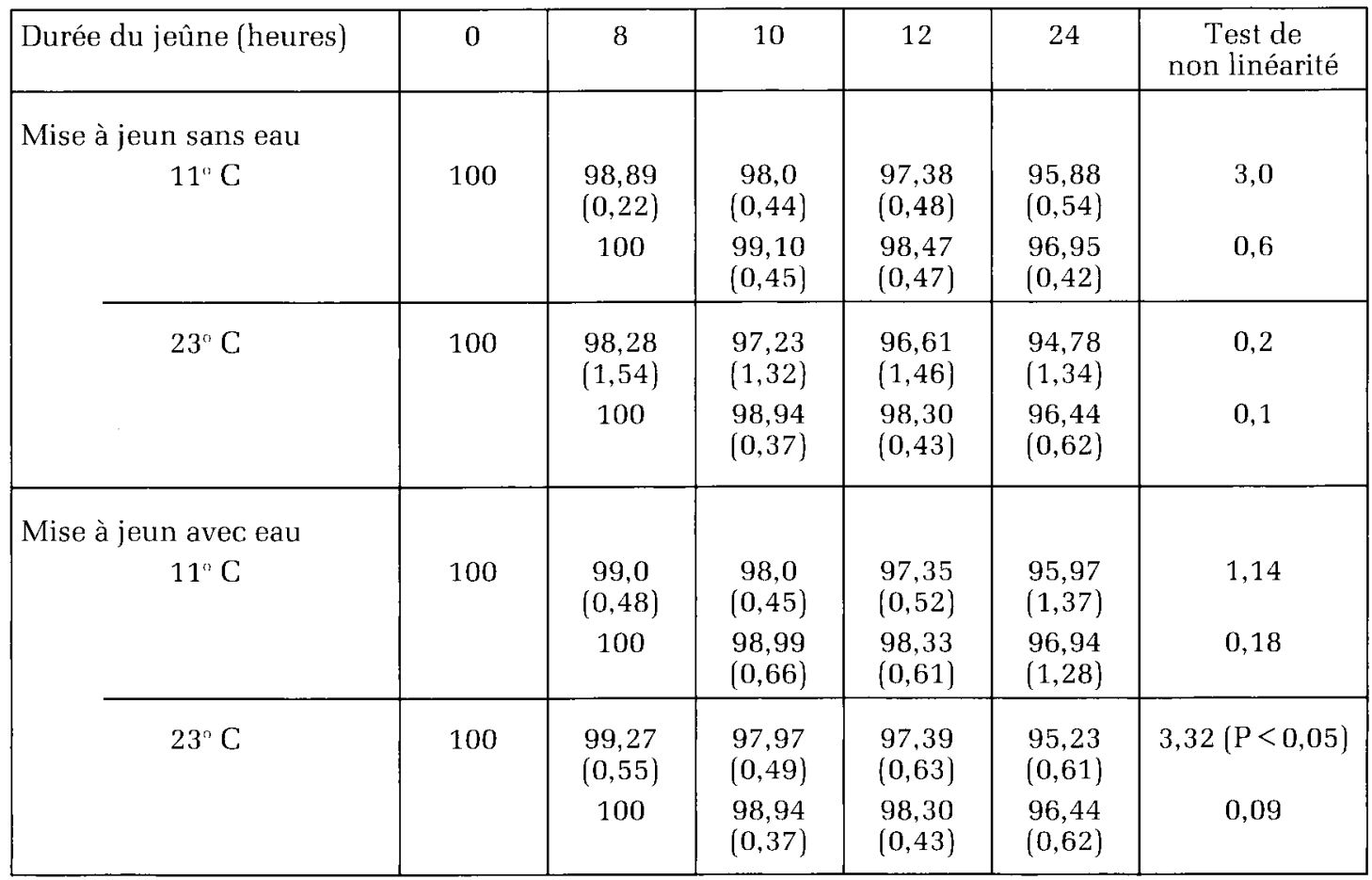

Entre parenthèses sont présentés les écarts-types. 
Figure 1. Evolution du poids vif du canard de Barbarie au cours du jeûne (expérience 1).

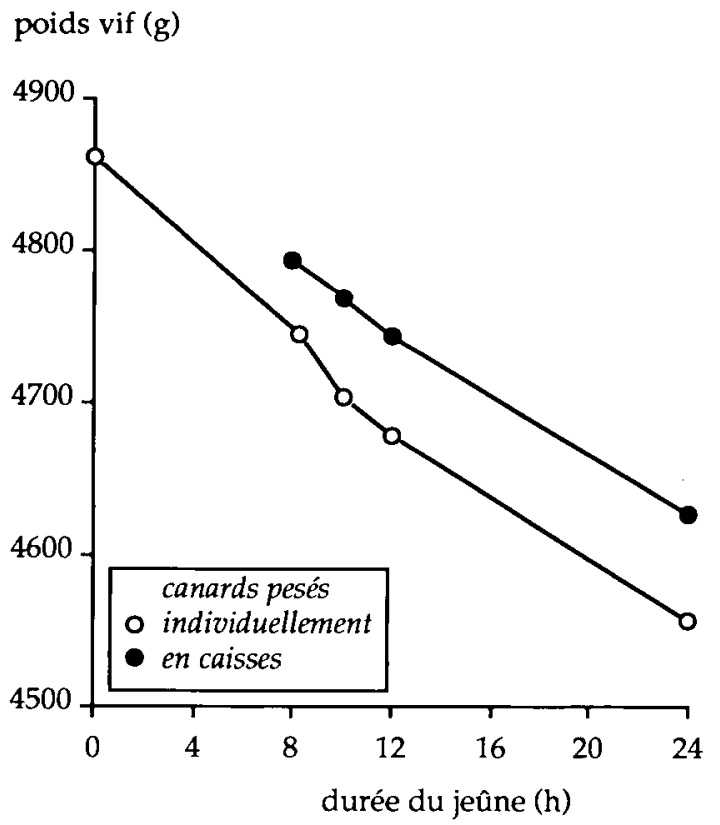

Pour faciliter les comparaisons entre traitements et entre expériences, les résultats ont aussi été calculés en valeur relative en prenant comme référence soit le poids vif à la mise à jeun, soit le poids à la mise en cage. Les premières font l'objet du tableau 3 . Si l'on ne considère que les régressions linéaires, éliminant le terme quadratique lorsqu'il existe, on remarque une forte différence de valeurs du coefficient de régression entre les deux expériences. On remarque aussi qu'au cours de la seconde expérience ce coefficient est plus élevé à $23^{\circ} \mathrm{C}$ qu'à $11^{\prime \prime} \mathrm{C}$. Lorsqu'on prend pour référence $(100)$ le poids à la mise en cage, c'est-àdire après 8 heures de jeûne (tableau 4), les droites de régression sont nettement plus reproductibles. En effet le coefficient linéaire de l'expérience 1 qui s'est déroulée à 18 " C est très proche de ceux de l'expérience 2 réalisée à $23^{\circ} \mathrm{C}$. Dans cette expérience la température de $11^{\circ} \mathrm{C}$ réduit la pente de décroissance du poids vif.

\section{Discussion et conclusion}

Ces deux expériences fournissent une estimation de la décroissance du poids vif entre la mise à jeun et l'abattage des animaux. La reproductibilité n'est pas très bonne quand on prend en considération les 8 premières heures de jeûne; ceci peut être dû aux conditions différentes de mise à jeun (âge des animaux, élevage sur litière ou grillage), à l'exception de l'abreuvement qui ne semble pas avoir d'effet d'après la seconde expérience.

En revanche, après la mise en cage, les pertes de poids vif sont très reproductibles d'un essai à l'autre. Elles sont un peu influencées par la

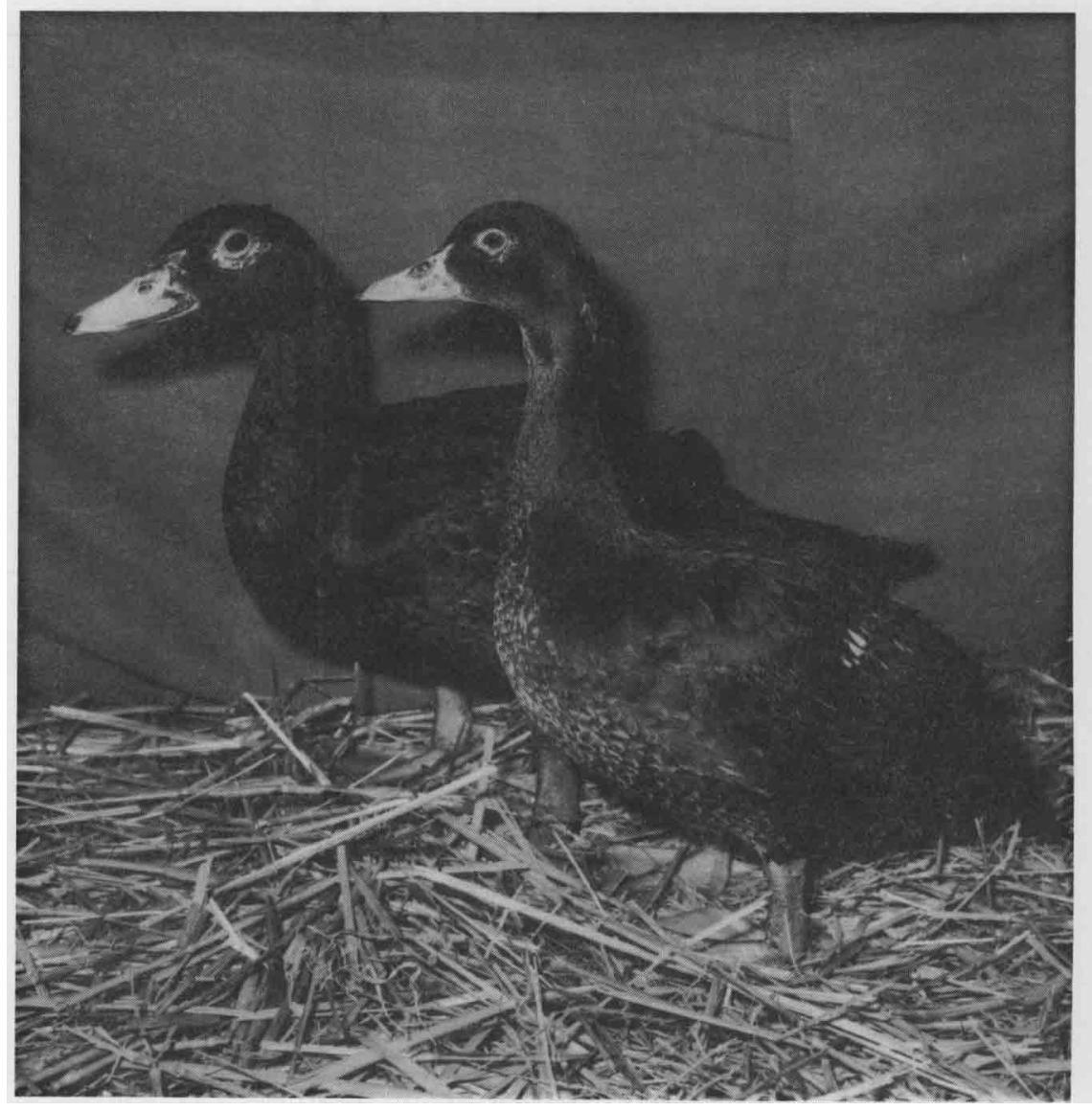

Figure 2. Evolution du poids vif du canard de Barbarie au cours du jeûne en fonction de la température ambiante et de l'abreuvement (expérience 2).

poids vif $(g)$

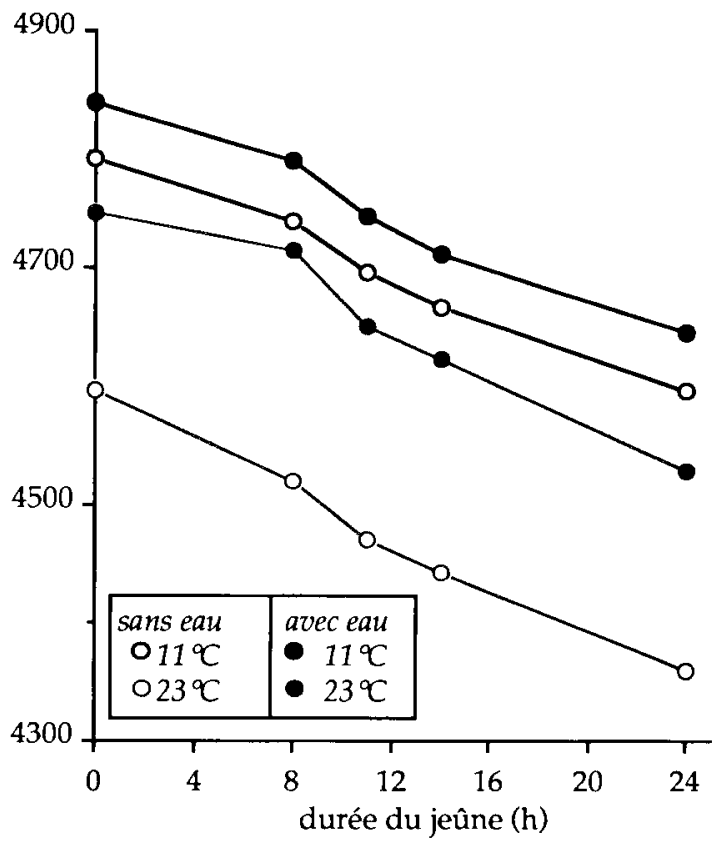


Tableau 3. Equations décrivant l'évolution du poids par rapport au poids de la mise à jeun.

\begin{tabular}{|c|c|c|c|c|c|}
\hline & Constante & $\begin{array}{c}\text { Coefficient } \\
\text { linéaire }\end{array}$ & $\begin{array}{c}\text { Coefficient } \\
\text { quadratique }\end{array}$ & R2 & $\begin{array}{c}\text { Variance } \\
\text { résiduelle }\end{array}$ \\
\hline Expérience 1 & 99,62 & $-0,259$ & & & \\
& 100,02 & $-0,367$ & $+0,0043$ & 0,940 & 0,264 \\
Expérience 2 & & & & & \\
sans eau & 100,04 & $-0,176$ & & 0,180 \\
$11^{\circ} \mathrm{C}$ & 99,89 & $-0,220$ & & 0,678 & 0,182 \\
$23^{\circ} \mathrm{C}$ & & & & & 1,504 \\
avec eau & 100,05 & $-0,174$ & & 0,792 & 0,214 \\
$11^{\circ} \mathrm{C}$ & 100,33 & $-0,208$ & $-0,0026$ & 0,897 & 0,313 \\
$23^{\circ} \mathrm{C}$ & 100,11 & $-0,145$ & $-0,908$ & 0,288 \\
\hline
\end{tabular}

Tableau 4. Equations décrivant l'évolution du poids par rapport au poids de la mise en caisse.

\begin{tabular}{|c|c|c|c|c|c|}
\hline & Constante & $\begin{array}{c}\text { Coefficient } \\
\text { linéaire }\end{array}$ & $\begin{array}{c}\text { Coefficient } \\
\text { quadratique }\end{array}$ & $\mathrm{R} 2$ & $\begin{array}{l}\text { Variance } \\
\text { résiduelle }\end{array}$ \\
\hline Expérience 1 & & & \multirow{11}{*}{$+0,0074$} & & \\
\hline Pesée & 99,80 & $-0,233$ & & 0,934 & 0,153 \\
\hline individuelle & 99,98 & $-0,360$ & & 0,943 & 0,134 \\
\hline Pesée & & & & & \\
\hline en caisses & 99,96 & $-0,217$ & & 0,982 & 0,037 \\
\hline \multicolumn{5}{|l|}{ Expérience 2} & \\
\hline $\begin{array}{l}\text { sans eau } \\
11^{\circ} \mathrm{C}\end{array}$ & 99.77 & -0.183 & & 0.895 & 0,149 \\
\hline $23^{\circ} \mathrm{C}$ & 99,75 & $-0,213$ & & 0,896 & 0,206 \\
\hline \multicolumn{5}{|l|}{ avec eau } & \\
\hline $11^{\circ} \mathrm{C}$ & 99,69 & $-0,181$ & & 0,672 & 0,622 \\
\hline $23^{\circ} \mathrm{C}$ & 99,64 & $-0,231$ & & 0,935 & 0,140 \\
\hline
\end{tabular}

température ambiante; la température la plus élevée produisant les pertes les plus importantes. Cet effet de la température mériterait d'être analysé en comparant plusieurs températures de façon à définir la fonction qui lie ces deux paramètres.

Le fait d'estimer le poids des animaux d'après le poids des cages en début de mise en cage sous-estime un peu la perte de poids ; ceci s'explique par le fait que les excreta que les oiseaux produisent encore après 8 heures de jeûne sont comptabilisés avec les animaux. Cet effet est toutefois très faible.

Quoi qu'il en soit on peut estimer la perte de poids au cours du séjour en cage de transport à $0,20 \%$ du poids à la mise en cage par heure de jeûne. Cette perte est un peu supérieure pour une température voisine de $20^{\circ} \mathrm{C}$ et un peu inférieure aux environs de $10^{\circ} \mathrm{C}$. L'intensité de ces pertes en fonction du temps est du même ordre que celle observée chez le poulet: de 0,20 à $0,24 \%$ par heure (Veerkamp 1985). D'autres facteurs mériteraient probablement d'être pris en considération. Cependant les résultats présentés ci-dessus fournissent un ordre de grandeur des pertes dues au jeûne chez le canard et suggèrent qu'elles sont assez semblables à celles du poulet en valeur relative.

\section{Référence bibliographique}

VEERKAMP C.H., 1985. Weight losses and yield after feed or feed and water withdrawal of broilers. In : Proceedings of the VIIth European Symposium on Meat quality, VEIJLE, Denmark 